\title{
Outsourcing in silico predictive toxicology: technologies, markets and paradigms
}

"This article provides a brief overview of the current and anticipated paradigms in drug development and examines how the adoption of breakthrough in silico predictive toxicology can play a key role in making the transition between the two."

Keywords: toxicity $\approx$ drug development $₫$ outsourcing $"$ predictive toxicology $\approx$ in silico

It has become a truism that the drug-development industry is in need of a new paradigm. Several publications detail this new business model and its effect on the therapeutic and financial potential of new drugs [1]. While not everyone agrees on the conclusions, the arguments are based on relevant and provable factors. The biggest of these are declining productivity and increasing regulatory restrictions. Together, they create a situation where there are fewer drugs in the development pipeline, and they all become more expensive to develop [2].

Interestingly, declining productivity and regulatory restrictions have something in common: both are rooted in predicting and characterizing toxicity. We will see how advances in this specialty can enable a transition to a new paradigm of drug development. Here, I will focus on the case of outsourcing predictive toxicology, particularly in silico methods. Outsourcing this particular service will be a case where we can see the challenges - and rewards - for extending the outsourcing model to this key discipline.

\section{Predictive toxicology: the pivot between paradigms}

Toxicity that is not discovered until after market release is a scenario all too common in the prescription drug market. This is a characteristic outcome of the current drug-development paradigm and it can be explained by looking at a schematic of the process as it occurs today (FIgUre I). This paradigm includes many steps, which have the basic goal of predicting the biological activity of compounds (efficacy and toxicity). In practice, the most relevant data usually come from clinical trials - and at great expense. Due to this, the clinical trial phase can be the key to a drug's success or the source of its demise. While this is not the most cost-effective way of identifying and characterizing toxicity, it is the de facto gold standard, for two reasons: regulatory and scientific necessity.

Current predictive toxicology models are not sufficiently predictive to match clinical trial data. While predictive toxicology certainly cannot replace any clinical trials, it has the potential to rationalize the process and increase its productivity. Avoiding futile entries into the clinical trial phase by early-stage elimination can save millions of dollars. Predictive toxicology can serve as the 'gate-keeper' to clinical trials.

The reason that late-stage discovery of toxicity is a characteristic of this model is visible in the placement of 'confidence in mechanism' (CIM) and 'confidence in safety' (CIS). CIM and CIS are not determined until the middle of clinical trials well after R\&D and therefore well after it is possible to easily modify the compound. The mechanistic knowledge that underlies CIM and CIS is invaluable for characterizing the potential toxicity of a compound. However, this information does not appear in time to make an impact on the $R \& D$ phase.

Establishing mechanistic knowledge early on is the first step to changing these results. A very direct application of mechanistic knowledge is the characterization of potential toxicity. Technological advances in this field will enable clinical trials to be initiated much more selectively. They can be confidently reserved for the compounds that have had the best characterization and stand the best chance of success on the market.

The appearance of this information within the $R \& D$ phase enables major changes to be made to the process. This change is clearly visible in the anticipated new paradigm of drug development (FIGURE 2).

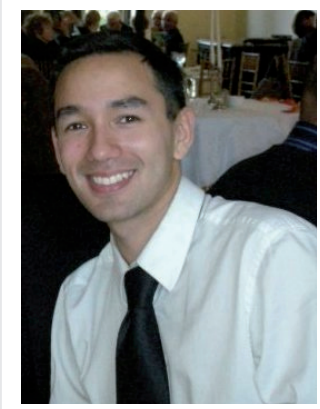

\section{Alexandre Ismail}

BioQuanta, 5 rue de l'Abbé de l'Epée, 75005 Paris, France Tel.: + 33950739326 Fax: $+33 \quad 14633 \mid 424$ E-mail: alexandre.ismail@ bioquanta.net 


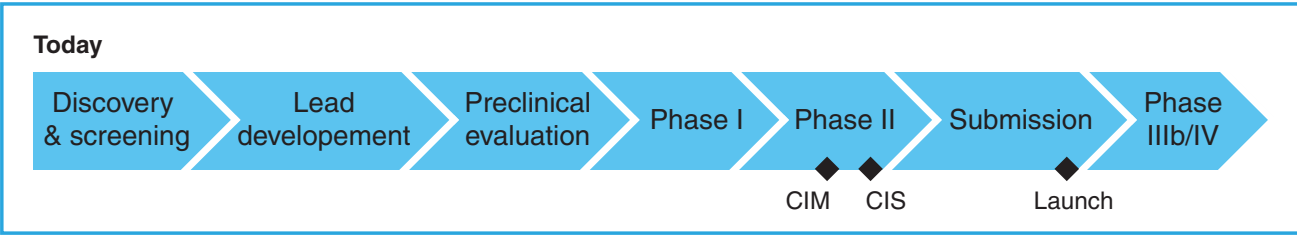

Figure 1. The current drug-development paradigm.

CIM: Confidence in mechanism; CIS: Confidence in safety.

Reproduced with permission from [1] (C) PriceWaterhouseCoopers.

We can see that this model of drug development is much more open to a market structure of outsourced service providers. 'Molecule development', which corresponds to 'lead development' in Figure I, has been broken down into several welldefined (and quite possibly professionally separate) specialties, with feedback loops explicitly present in their interactions. Many of these tasks could be the work of an outsourced specialist or they could be retained as internal activities of the larger drug developer. The larger drug developer can still chose which activities to outsource, and can explore outsourcing options in a gradual way. This will allow the effective 'sampling' of innovative approaches without losing the infrastructure and organization required to manage clinical trials.

It is also important to note that two previously sequential and widely separated control points, pharmaceutical sciences and regulatory toxicology, have now become parallelized. These are two major challenges of today's market landscape and their importance will only increase. Yet they need no longer be seen as barriers. The ability to have rapid and early feedback between these two elements enables drug developers to refine leads more precisely through focused iterations of optimization in $\mathrm{R} \& \mathrm{D}$; this ultimately depends on a mechanistic understanding of molecular action. This is why a mastery of molecular modeling is a technological and functional necessity of the new model. The main application of this discipline is known as in silico predictive toxicology, and it is strategically positioned to be an enabling technology for the new paradigm.

\section{Predictive toxicology: in silico, outsourced}

So far we have reviewed the role of predictive toxicology as part of the bigger picture of development paradigms. We can now focus on the specific challenges in silico predictive toxicology will have to face - especially if it is outsourced.

The main challenge is maintaining the confidentiality of the chemical entity in development. Understandably, this constraint means that most preliminary characterizations of efficacy and toxicity are performed in-house. This situation seems obvious and necessary, yet it contains one key assumption and one key problem, both of which are amenable to technological advances.

The key problem is that many in silico toxicity prediction systems have reached a plateau of predictive power and practical utility. Developments in the field have progressed quite uniformly and, despite being run in-house, many toxicity predictions approaches are quite similar in their fundamental mechanics. To achieve the kind of performance necessary to enable paradigm changes, innovation is clearly needed - specifically in the field of molecular modeling. Such a solution is most likely to come from a specialist, and such a specialist would likely provide their technology as an outsourced service.

Outsourcing this specialty implies sharing data regarding the chemical entity in development. This brings us to the key assumption, which is that predicting the toxicity of a given compound requires its structure to be disclosed. Recent advances in molecular modeling show that this is no longer the case [3]. Rethinking the fundamentals of molecular modeling has led to the development of new technology. If we admit that it is possible to predict toxicity without disclosure of the chemical entity, then we have overcome the main challenge to outsourcing this key discipline.

There are several advantages to this situation. Some of these are direct short-term advantages resulting from the use of such a technology. Enhanced molecular design can impact any given drug developer in a couple of ways, depending on how it is deployed: as a toxicity-screening service or as a more complete lead-optimization service.

A toxicity-screening approach that is able to identify toxic drug candidates early makes it possible to eliminate them early. This will have the effect of increasing preclinical attrition rates, while ultimately decreasing post-clinical attrition rates. Increasing the preclinical attrition rate may seem unattractive at first, but it is precisely 
by being more discriminating at the beginning that more money is saved at the end. A leadoptimization approach is the complement to screening - instead of weeding out problematic molecules, a better molecule can be designed. Outsourcing also brings the usual advantages of not having to invest in the infrastructure and personnel for a given service.

There are some additional indirect advantages that will benefit the industry at large, and these stem from a more open market structure of outsourced specialists. One is competition, which will stimulate innovation in a field that clearly needs techniques beyond statistical correlation quantitative structure-activity relationships [4]. Competition inevitably rests on comparison of results, and this can be facilitated by the development of industry standards. Standards for the accuracy of in silico predictions will also be of great help in regulatory compliance, since several recent regulations have placed limits on animal testing.

Many of the elements we have discussed so far seem to be relevant to future scenarios. However, it is the near future, probably within 5-10 years, and that means that the transition period is now. The regulatory and productivity constraints that we have seen appear over the past few years will be the driving force of the paradigm change, and better molecular modeling will be the means of implementing it.

Financial and regulatory pressures have helped place a premium on mechanistic understanding [101], and so the predictive toxicology services market is one that posseses major growth potential over the coming years - especially for those who can overcome the technological challenge of maintaining new chemical entity confidentiality. Since many more industry players are now in need of toxicity data, we will likely see a diversification of customers (according to the specific demand driver) as well as solutions to meet their needs. The future will continue to bring a few breakthrough innovations against a backdrop of incremental improvements to existing technology, with each specializing in a particular area of demand.

There is still a long way to go to integrate these in silico solutions into the big pharmaceutical population at large. Toxicity prediction is a clear-cut application and a good one to start with. It also opens the door for the more complete chemical optimization with more extensive use of these techniques [5].

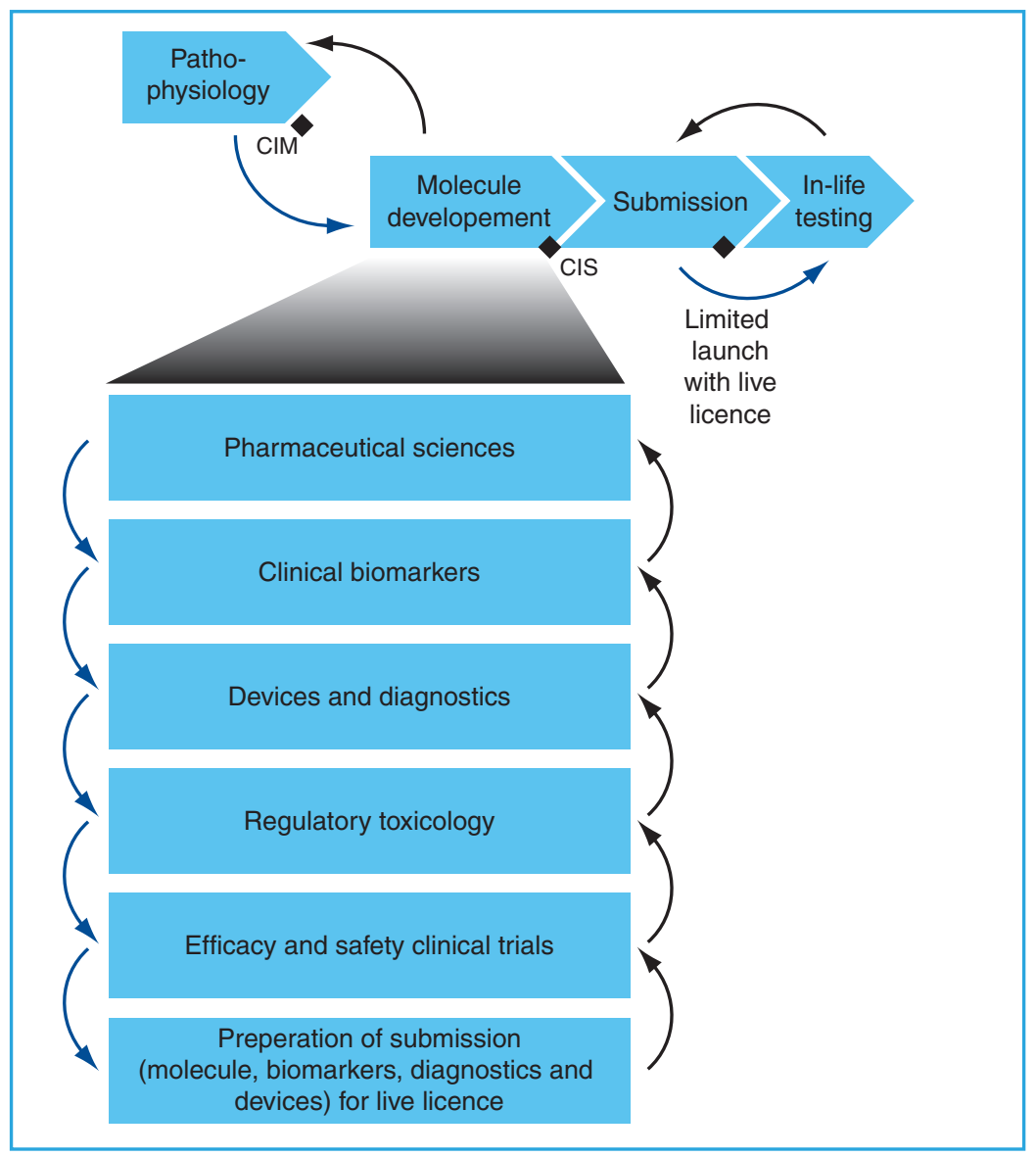

Figure 2. Proposed new drug-development paradigm.

CIM: Confidence in mechanism; CIS: Confidence in safety.

Reproduced with permission from [1] () PriceWaterhouseCoopers.

Mechanism-based modeling will also be expanded from the molecule to the patient, in the form of increasingly detailed absorption, distribution, metabolism, excretion and toxicity simulations. As biochemical modeling becomes a more mature technology, it can provide the additional information necessary to support new types of therapy. A comprehensive integration of such in silico tools for reliable decision support can eventually be extended to personalized medicine, which requires a rapid and comprehensive biochemical characterization of individual patients.

\section{Financial \& competing interests disclosure}

The author is currently employed by BioQuanta, which provides outsourced services in predictive toxicology. The author has no other relevant affliations or financial involvement with any organization or entity with a financial interest in or financial conflict with the subject matter or materials discussed in the manuscript apart from those disclosed.

No writing assistance was utilized in the production of this manuscript. 


\section{EDITORIAL | Ismail}

\section{Bibliography}

1 PriceWaterhouseCoopers Analysis. Pharma 2020: The Vision - Which Path Will You Take? PriceWaterhouseCoopers, London, UK, June 2007.

2 DiMasi JA. The value of improving the productivity of the drug development process: faster times and better decisions.

Pharmacoeconomics 20(Suppl. 3), 1-10 (2002).
3 Guerin GA, Pratuangdejkul J, Alemany M, Launay JM, Manivet P. Rational and efficient geometric defintion of pharmacophores is essential for the patent process. Drug Discov. Today 11(21-22), 991-998 (2006).

4 Maggiora GM. On outliers and activity cliffs - why QSAR often disappoints. J. Chem. Inf. Model. 46, 1535 (2006).

5 Peters MB, Raha K, Merz KM Jr. Quantum mechanics in structure-based drug design. Curr. Opin. Drug Discov. Devel. 9(3), 370-379 (2006).

\section{- Website}

101 REACH legislation: Annex XV Restriction Report - Proposal for a restriction of dimethyl fumarate - by ANSES (The French agency for food, environmental, and occupational health safety).

http://echa.europa.eu/doc/restrictions/ annex_xv_restriction_report_DMFu_en.pdf 